${ }^{1}$ Department of Orthodontics, Faculty of Dentistry, Sinai University, Kantara branch.

${ }^{2}$ Associate professor, Department of Orthodontics and Pediatric Dentistry, National Research Centre.
Corresponding author: Dr. Omnia A. Elhiny, National Research Centre, Egypt, 33 El Bohouth St., Dokki, Giza, P.O. 12622, Egypt.

Phone: +2 01112826250 Fax: +2 0233370931

Email:omniaelhiny@yahoo.com

Editor: Altair A. Del Bel Cury

Received: November 02, 2020

Accepted: August 20, 2021

\section{Evaluation of an open access generic 3D software for Orthodontic diagnosis and treatment planning}

\author{
Hany Salah Eldin Eid ${ }^{1}$, Omnia A. Elhiny ${ }^{2 \star}$
}

Aim: To evaluate the accuracy and the validity of orthodontic diagnostic measurements, as well as virtual tooth transformations using a generic open access 3D software compared to OrthoAnalyzer (3Shape) software; which was previously tested and proven for accuracy. Methods: 40 maxillary and mandibular single arch study models were duplicated and scanned using 3Shape laser scanner. The files were imported into the generic and OrthoAnalyzer software programs; where linear measurements were taken twice to investigate the accuracy of the program. To test the accuracy of the program format, they were printed, rescanned and imported into OrthAnalyzer. Finally, to investigate the accuracy of editing capabilities, linear and angular transformation procedures were performed, superimposed and printed to be rescanned and imported to OrthoAnalyzer for comparison. Results: There was no statistically significant difference between the two groups using the two software programs regarding the accuracy of the linear measurements $(p>0.05)$. There was no statistically significant difference between the different formats among all the measurements, ( $p>0.05)$. The editing capabilities also showed no statistically significant difference $(p>0.05)$. Conclusion: The generic 3D software (Meshmixer) was valid and accurate in cast measurements and linear and angular editing procedures. It can be used for orthodontic diagnosis and treatment planning without added costs.

Keywords: Software. Imaging, three-dimensional. Dental models. Casts, surgical. 


\section{Introduction}

Orthodontists are currently facing the challenge of continuous technological advancements in computer sciences. Digitization in orthodontics has transformed from digital records and images into digital study casts, digital analysis, digital virtual tooth movements and fabrication of Aligners and appliances ${ }^{1,2}$.

In addition, the availability of 3D orthodontic software programs has facilitated the study of maxillary, mandibular, intra-arch and inter-arch relations, as well as performing virtual setups and treatment plans ${ }^{1,3,4}$.

Study models are crucial for orthodontic diagnosis and treatment planning. The recent use of digital casts has allowed for a wider scale of communication between orthodontists and easier marketing to attract patients without the need for a physical storage space ${ }^{5}$.

In early 1999, the first digital system was introduced and nowadays many systems are commercially available ${ }^{6}$. This presence of different technologies calls for a proper standardization and the investigation of the accuracy of digital measurements compared to those on plaster study models. Many systems were evaluated; such as Digimodel, ${ }^{4}$ O3DM ${ }^{7}$, OrthoCAD ${ }^{1}$ and 3shape OrthoAnalyzer ${ }^{2,5,6}$, and were found to be as reliable and accurate as plaster models; which means that digital models can be considered as the new gold standard ${ }^{5,8-10}$.

There are increasing office expenses and training that have to be met in order to gain maximum benefits using such a fast-developing technology. Hence, the presence of a free software; that is easy to operate and accessible for download on the web, appears to be an appealing alternative to many. The question lies here in whether this software program is valid and accurate or not.

The aim of this study was to evaluate the accuracy and the validity of orthodontic diagnostic measurements, as well as virtual tooth transformations using a generic open access 3D software compared to OrthoAnalyzer (3Shape) software; which was previously tested and proven for accuracy.

\section{Material and methods}

Forty single arch sets of maxillary and mandibular study models were included in the study. The sample size calculation showed that this number provided a statistical power of $80 \%, a=0.05$ and $\beta=0.20$.

The study models were duplicated from those of patients attending a private orthodontic practice. The selection criteria were intact full set of permanent dentition from permanent first molar to permanent first molar upper and lower. No previous orthodontic treatment, no gingival inflammation or recession, no dental attrition or erosion, and no crowding or mild to moderate crowding (not more than $4 \mathrm{~mm}$ ) and no voids or blebs in the stone or digital models.

The models were duplicated using Jeltrate fast-set alginate (Sirona Dentsply) in stock impression trays and poured into type IV dental stone (GC FUJIROCK EP Polar White, 
GC Europe N.V, Belgium). Digital impressions were recorded using laser scanner (3Shape D500, 3Shape A/S, Copenhagen, Denmark). The specifications and accuracy of the laser scanner have been validated in previous studies ${ }^{11}$. After scanning all the sample by the laser scanner, default STL files (Standard Triangle Language) were created and imported one by one on Microsoft Windows 7 desktop computer to both software programs; generic open access software (Autodesk Meshmixer 3.5 for windows, USA) and OrthoAnalyzer (version 1.5; 3Shape, Copenhagen, Denmark). Once imported, the formats of scanned models were changed automatically to its corresponding format specific to the software.

The investigation was then conducted in three steps. Firstly, linear measurements were made twice on the scanned images to investigate the accuracy of the generic software. The first measurements were done using the Meshmixer; those measurements were given the symbol GM; Figure 1a. The second measurements were done using OrthoAnalyzer, and those measurements were given the symbol SM1;

Figure $\mathbf{1 b}$. The OrthoAnalyzer measurements were considered as control since their accuracy and validity were established in previous studies ${ }^{1,2,6,7,12,13}$. All the measurements were performed by the same investigator.

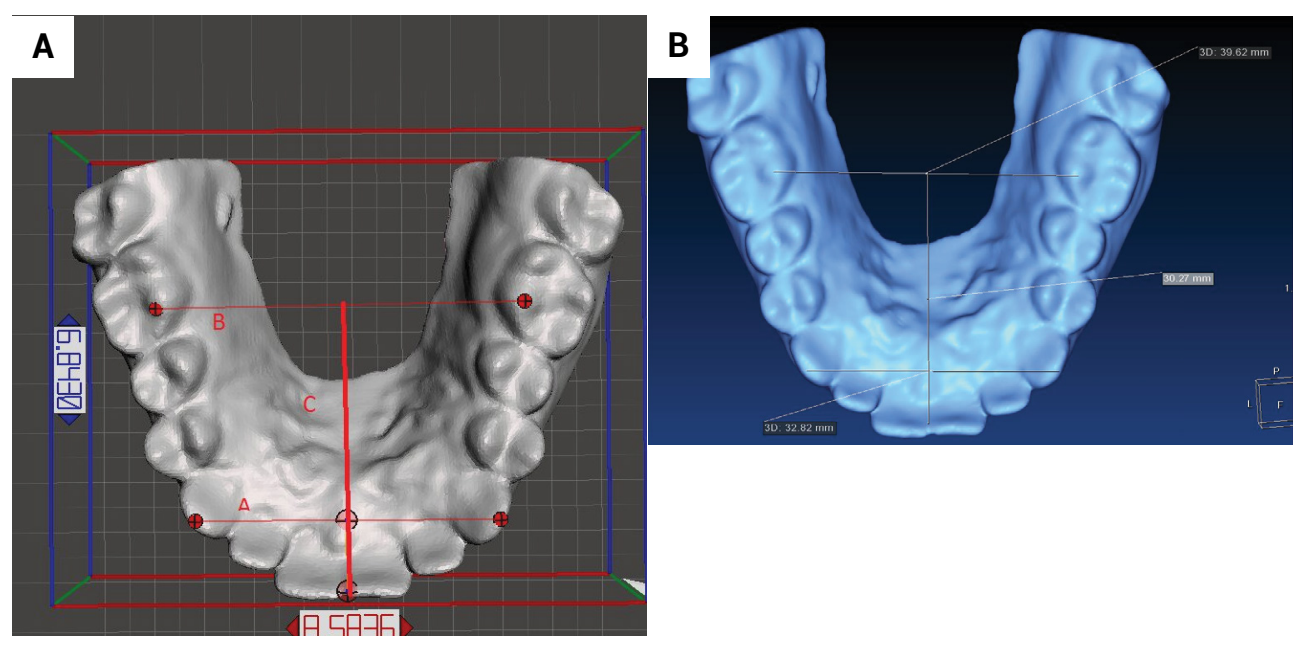

Figure 1. A - Meshmixer measurements GM. B - OrthoAnalyzer measurements SM1.

The linear measurements taken were:

$A=$ Intercanine width: the distance between the cusp tips.

$B=$ Intermolar width: the distance between the mesiopalatal cusp tips of maxillary first permanent molars. The distance between the mesiobuccal cusp tips of mandibular first permanent molars.

$\mathrm{C}=$ Arch depth: the perpendicular distance from the incisal edge at the midline to the mesiobuccal cusp tip of mandibular molars and the mesiopalatal cusp tip for maxillary molars. 
Secondly, the accuracy of the generic software format was tested where the scanned models with the Meshmixer format (generic software format) were exported to a digital printer (Formlabs, Somerville, MA, USA). They were retransformed into physical models which were rescanned and a second STL file was created, imported and reanalyzed using the OrthoAnalyzer (SM2). After that, SM1 and SM2 measurements were analyzed to compare the stability of the initial model measurements (SM1) to those taken after the models' format was changed from STL to Meshmixer format and then again to the second STL and OrthoAnalyzer format (SM2).

Thirdly, the accuracy of the editing capabilities of the generic software was tested using copies of all the original models. The copies were saved on both software programs and were subjected to two editing transformation procedures on two anterior teeth; one movement per tooth. Using the generic software, the first movement was an unspecified labial tipping movement not exceeding the labiolingual thickness of that particular tooth. The second movement was a 5-degree labial rotation around the mesial axis of the tooth. The edited model copies were then superimposed over the original models of the same case. The amount of labial linear transformation for the first tooth (GT1) and the linear transformation of the distal contact point of the second tooth, as a result of tooth rotation around its mesial axis (GT2), were measured; Figure 2a. Both movements were exported and printed in the superimposed form. This printed form was rescanned and imported to the OrthoAnalyzer software

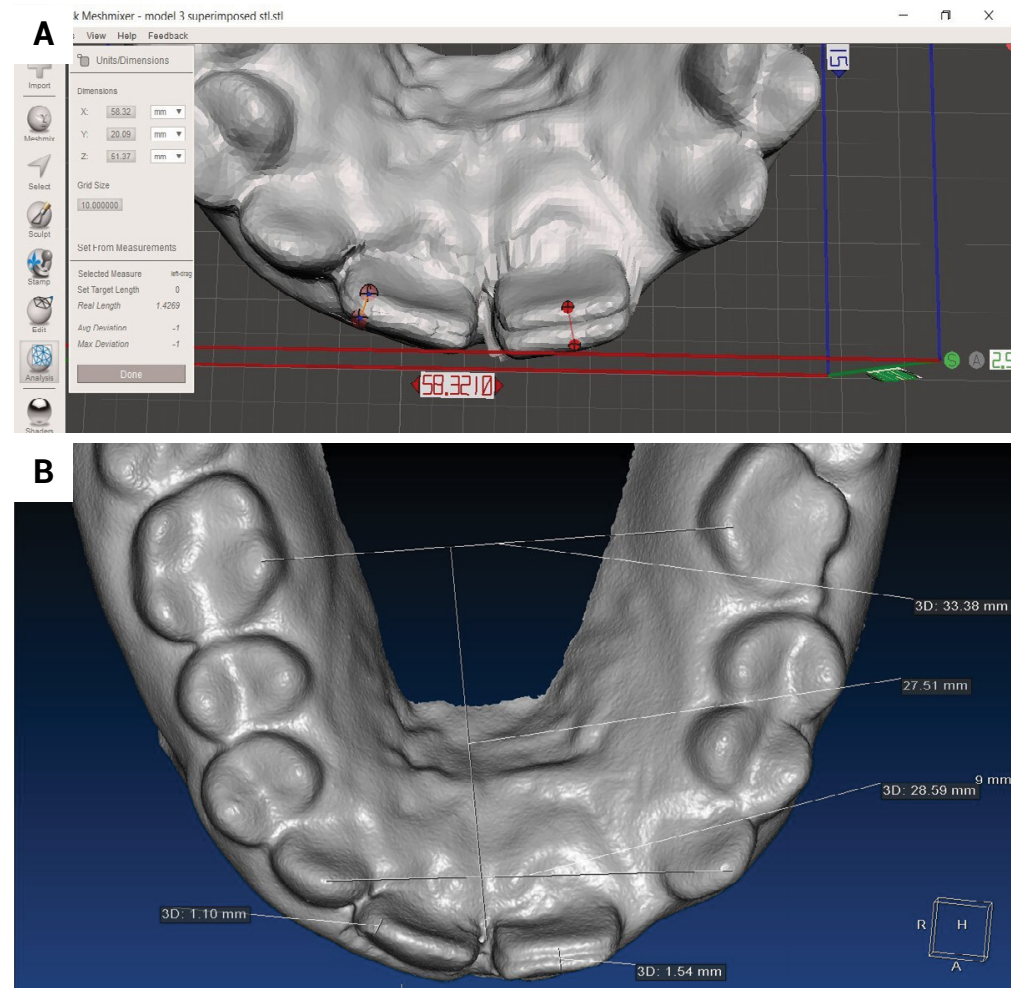

Figure 2. A - Meshmixer measurement of the amount of labial linear transformation of the first tooth and linear transformation of distal contact point of the second tooth; GT1 and GT2. B - The same measurements as in A, but using OrthoAnalyzer; ST1 and ST2. 
where the same measurements were performed (ST1 \& ST2); Figure $2 \mathbf{b}$. Measurements from both software programs were then compared.

The mean and standard deviation values were calculated for each group in each test. Data were explored for normality using Kolmogorov-Smirnov and Shapiro-Wilk tests and showed parametric (normal) distribution. Paired sample t-test was used to compare between two groups in related samples. The significance level was set at $P \leq 0.05$. Statistical analysis was performed with IBM® SPSS ${ }^{\circledR}$ Statistics Version 20 for Windows.

\section{Results}

I. Measuring the accuracy of generic software to perform measurements on scanned models GM (GM vs SM1):

The accuracy of generic software to perform measurements is represented in

Figure 3. The means and standard deviations of GM vs SM1 are shown in Table 1.

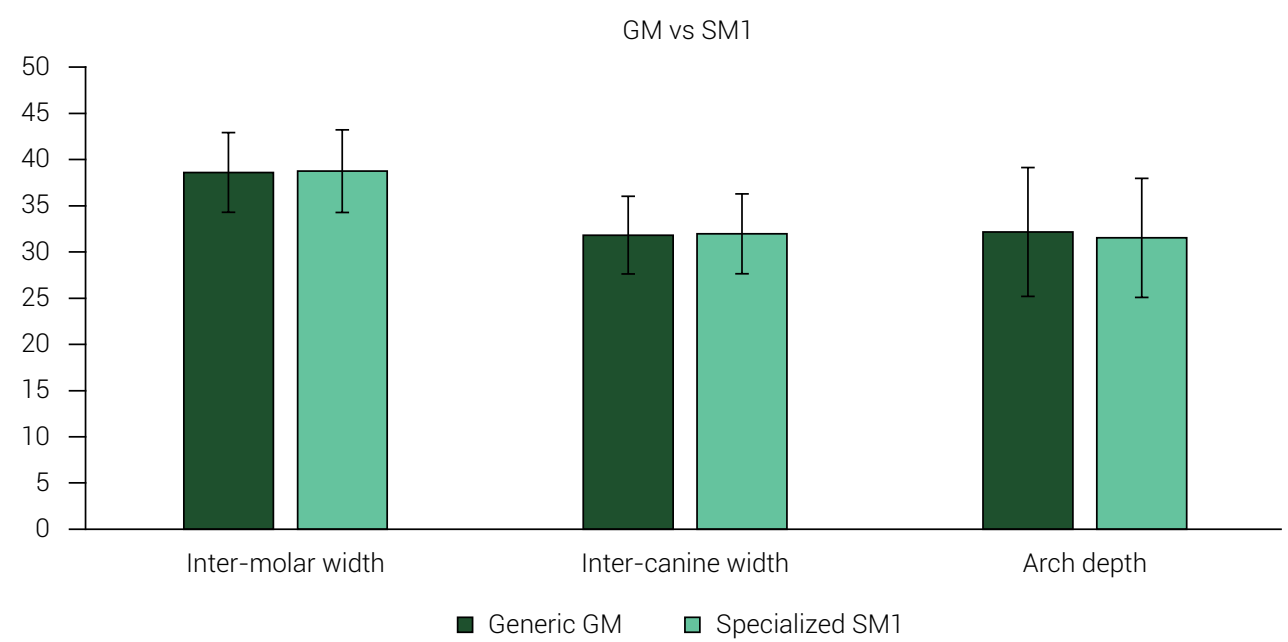

Figure 3. Bar chart representing GM vs SM1.

Table 1. Mean and standard deviation (SD) values of GM vs SM1

\begin{tabular}{llllll}
\hline \multirow{2}{*}{ Variables } & \multicolumn{5}{c}{ GM vs SM1 } \\
\cline { 2 - 5 } & \multicolumn{2}{c}{ Generic GM } & \multicolumn{2}{c}{ Specialized SM1 } & \multirow{2}{*}{ p-value } \\
\cline { 2 - 5 } & Mean & SD & Mean & SD & \\
\hline Inter-molar width & 38.60 & 4.31 & 38.74 & 4.47 & $\mathbf{0 . 6 3 5 n s}$ \\
\hline Inter-canine width & 31.82 & 4.20 & 31.96 & 4.32 & $\mathbf{0 . 2 6 3 n s}$ \\
\hline Arch depth & 32.16 & 6.97 & 31.52 & 6.44 & $\mathbf{0 . 1 3 7 n s}$ \\
\hline
\end{tabular}

ns; non-significant $(p>0.05)$

1. Inter-molar width:

There was no statistically significant difference between (Generic GM) and (Specialized SM1) groups where $(p=0.635)$. 
The highest mean value was found in (Specialized SM1) group, while the lowest mean value was found in (Generic GM) group.

2. Inter-canine width:

There was no statistically significant difference between (Generic GM) and (Specialized SM1) groups where $(p=0.263)$.

The highest mean value was found in (Specialized SM1) group, while the lowest mean value was found in (Generic GM) group.

3. Arch depth:

There was no statistically significant difference between (Generic GM) and (Specialized SM1) groups where $(p=0.137)$.

The highest mean value was found in (Specialized SM1) group, while the lowest mean value was found in (Generic GM) group.

I. Measuring the accuracy of original cast analysis measurements after changing the scanned models' format to generic software format SM (SM1 vs SM2):

The accuracy of the original cast analysis measurements is represented in Figure 4. The means and standard deviations of SM1 vs SM2 are shown in Table 2.

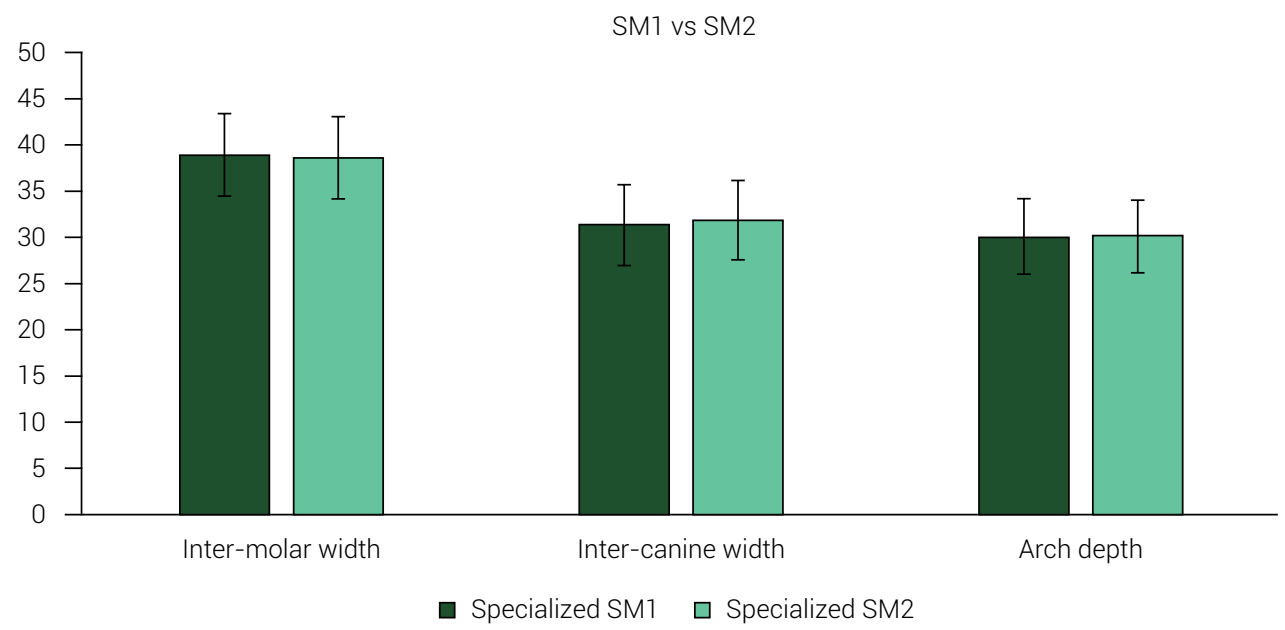

Figure 4. Bar chart representing SM1 vs SM2.

Table 2. Mean and standard deviation (SD) values of SM1 vs SM2

\begin{tabular}{llllll}
\hline \multirow{2}{*}{ Variables } & \multicolumn{4}{c}{ SM1 vs SM2 } \\
\cline { 2 - 5 } & \multicolumn{2}{c}{ Specialized SM1 } & \multicolumn{2}{c}{ Specialized SM2 } & \multirow{2}{*}{ p-value } \\
\cline { 2 - 5 } & Mean & SD & Mean & SD & \\
\hline Inter-molar width & 39.02 & 4.43 & 38.74 & 4.47 & $\mathbf{0 . 0 6 6 n s}$ \\
\hline Inter-canine width & 31.46 & 4.38 & 31.96 & 4.32 & $\mathbf{0 . 4 0 3 n s}$ \\
\hline Arch depth & 30.22 & 4.02 & 30.26 & 3.93 & $\mathbf{0 . 4 7 7 n s}$ \\
\hline
\end{tabular}

ns; non-significant $(p>0.05)$ 
1. Inter-molar width:

There was no statistically significant difference between (Specialized SM1) and (Specialized SM2) groups where $(p=0.066)$.

The highest mean value was found in (Specialized SM1) group, while the lowest mean value was found in (Specialized SM2) group.

2. Inter-canine width:

There was no statistically significant difference between (Specialized SM1) and (Specialized SM2) groups where $(p=0.403)$.

The highest mean value was found in (Specialized SM2) group, while the lowest mean value was found in (Specialized SM1) group.

3. Arch depth:

There was no statistically significant difference between (Specialized SM1) and (Specialized SM2) groups where $(p=0.477)$.

The highest mean value was found in (Specialized SM2) group, while the lowest mean value was found in (Specialized SM1) group.

I. Measuring the accuracy of generic software to perform editing transformation on scanned casts T (GT $1 \& 2$ vs ST 1\&2):

The accuracy of generic software to perform editing transformation is represented in Figure 5. The means and standard deviations of GT 1\&2 vS ST1\&2 are shown in Table 3.

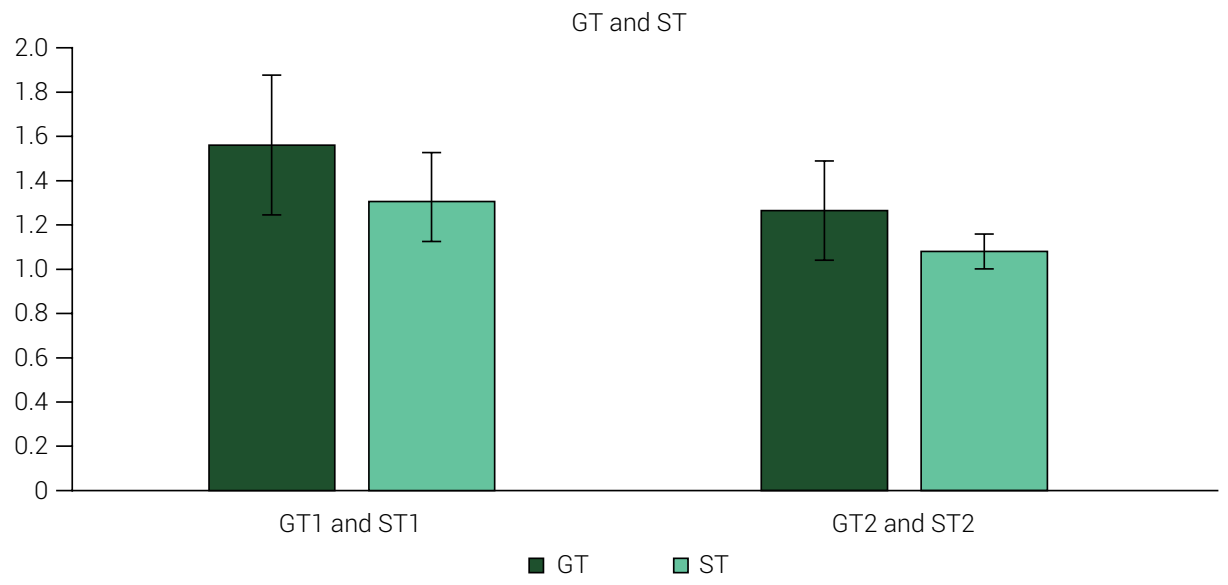

Figure 5. Bar chart representing GT $1 \& 2$ vs ST $1 \& 2$.

Table 3. Mean and standard deviation (SD) values of GT $1 \& 2$ vs ST $1 \& 2$

\begin{tabular}{lcccccccc}
\hline \multirow{2}{*}{ Variables } & \multicolumn{3}{c}{ GT1 } & \multicolumn{2}{c}{ ST1 } & \multicolumn{2}{c}{ GT2 } & \multicolumn{2}{c}{ ST2 } \\
\cline { 2 - 10 } & Mean & SD & Mean & SD & Mean & SD & Mean & SD \\
\hline GT 1\&2 vs ST 1\&2 & 1.56 & 0.31 & 1.32 & 0.20 & 1.26 & 0.23 & 1.08 & 0.08 \\
\hline p-value & \multicolumn{4}{c}{$\mathbf{0 . 1 1 8 n s}$} \\
\hline
\end{tabular}

ns; non-significant $(p>0.05)$ 
1. There was no statistically significant difference between (GT1) and (ST1) groups where $(p=0.118)$.

The highest mean value was found in (GT1) group, while the lowest mean value was found in (ST1) group.

2. There was no statistically significant difference between (GT2) and (ST2) groups where $(p=0.137)$.

The highest mean value was found in (GT2) group, while the lowest mean value was found in (ST2) group.

\section{Discussion}

It is an undeniable fact that we currently live in an electronic era where the utilization of virtual models has become increasingly important in the orthodontic practice ${ }^{2,14-17}$. Consequently, the availability of a well-established software has become crucial. This has been manifested by the abundance of a generous market; providing a rather noticeable number of well-established software programs, investigated for validity and accuracy, and capable of executing multifunctional tasks. However, most of those software programs are considered rather expensive, considerably complicated and require extensive training to master their potentials in full $4,18-20$, which restrains their use to a limited fraction of the realm of orthodontic practices. On the other hand, there is another domain of open access software programs available; not necessarily originally designed for dental work, and haven't been investigated for their potentials. The availability of such software programs provides a golden opportunity for enhancing the performance of the orthodontic clinic, have they been proven to be as effective as the market provided programs without the burden of the extensive finances.

Therefore, the present study compared the accuracy of a generic open access 3D software (Meshmixer) to that of OrthoAnalizer (version 1.5; 3Shape, Copenhagen, Denmark).

The investigation was conducted in three steps, where linear cast measurements' accuracy was compared between the two software programs; as the first step. There was no significant difference between the Meshmixer (GM) and 3Shape (SM1) measurements regarding the inter-molar and inter-canine widths as well as the arch depth which were needed on the diagnostic level of comparison. The dental cast measurements taken by the 3Shape OrthoAnalizer software are known from previous studies to be as accurate and valid as the measurements taken on plaster or stone dental

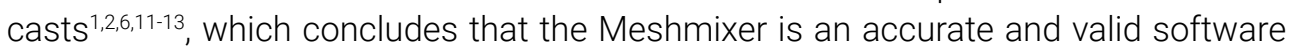
as a diagnostic measurement tool.

The investigated Meshmixer software was not designed specifically for orthodontic use and it is well known that the accuracy of orthodontic measurements is crucial to the diagnosis and treatment planning. This necessitated the investigation of the accuracy of the Meshmixer file format to determine the stability and reproducibility of its measurements ${ }^{12}$.

When the dental casts are scanned, they are normally stored as STL files and then imported to the analyzing software where they are converted into its suitable 
format ${ }^{10,11,21}$. The previously measured casts in the Meshmixer format (SM1) were exported and printed, then rescanned and imported as STL files into the 3Shape OrthoAnalizer software. Those files were then converted into the 3Shape format and the casts were then reanalyzed (SM2). When SM1 was compared to SM2, there was no significant difference which indicated that there was an accurate smooth conversion from the STL format to the Meshmixer format and comparable to the conversion provided by 3Shape OrthoAnalizer.

There is a worldwide increased demand for simulated treatment plans and customized orthodontic appliances for the sake of providing a more efficient and calculated treatment ${ }^{4}$.

Therefore, the virtual software used must be able to face this challenge by providing accurate angular and linear editing capabilities. This transformation potential is considered the corner stone in moving forward towards digital treatments. In this study, there was no statistically significant difference between the Meshmixer and the 3Shape in either of the linear or angular editing movements performed; which indicated that the Meshmixer was valid and accurate in performing the different editing movements. Most of the previous studies addressed the accuracy of the virtual measurements and model analysis, using software programs available in the market, and showed their comparability to the measurements on plaster models. Nonetheless, they seldom explored the software potential to accurately select and transform, digitally, a unit or multiple units within the dental model for correcting teeth positions. Further, they didn't investigate the accuracy of the file format for assessing the stability and reproducibility of the measurements of the used programs. This could be clearly seen in the studies conducted by Bukhari et al. ${ }^{6}$ where they investigated the accuracy of measurements of 3shape Ortho analyzer and Camardella et al. ${ }^{4}$ who compared the measurements of 3 Shape and Digimodel Orthoproof. Sousa et al. ${ }^{1}$ also examined the accuracy of Geometric Studio 5 software and Westerlund et al. ${ }^{5}$ compared 4 market software programs; Cadent, Ortholab, Orthoproof and 3 Shape. This drops the light on the importance of this investigation and its value in the future of digitization in Orthodontics.

The constant advances in the digital world have reduced the obstacles in diagnosis and treatment planning. The ability to plan treatment, design appliances and make treatment simulations and store every step for reference has escalated the need for such digital technology in every orthodontic practice. The main disadvantage of such technology discussed in the literature was the high costs and extensive training that come with it $4,18-20$. The Meshmixer software has proven to be a valid, accurate and reproducible measurement and editing tool; with the advantage of being an open access software on the internet that is easy to comprehend; such a finding will allow the delivery of a more efficient and predictable treatment for a wider base of patients.

On the other hand, this study was burdened by the limitation of the need to increase the vectors of the mechanical virtual movements performed on the units transformed; which should serve as an eye opener to software providers for making future improvements in their programs to address a wider base of users. 
Digital transformation has become the feature of today and not the future and the existence of such digital potentials, via open access programs, in one's hand represents a leap forward towards a new era of digital orthodontics. It also puts an additional responsibility on every operator to learn such new techniques as quickly as possible since their learning and tutoring has become, now, a must and not an additional luxury.

In conclusion, the generic software (Meshmixer) was accurate and valid in cast measurements, as well as linear and angular editing procedures. It provided stable and reproducible measurements. The generic software (Meshmixer) can be used in orthodontic diagnosis and treatment planning without any added costs for the orthodontist.

\section{References}

1. Sousa MVS, Vasconcelos EC, Janson G, Garib D, Pinzan A. Accuracy and reproducibility of 3-dimensional digital model measurements. Am J Orthod Dentofacial Orthop. 2012 Aug;142(2):269-73. doi: 10.1016/j.ajodo.2011.12.028.

2. Moreira DD, Gribel BF, Torres GDR, Vasconcelos KF, de Freitas DQ, Ambrosano GMB. Reliability of measurements on virtual models obtained from scanning of impressions and conventional plaster models. Braz J Oral Sci. 2014 Oct;13(4):297-302. doi: 10.1590/1677-3225v13n4a11.

3. Hajeer MY, Millett DT, Ayoub AF, Siebert JP. Applications of 3D imaging in orthodontics: part I. J Orthod. 2004 Mar;31(1):62-70. doi: 10.1179/146531204225011346.

4. Camardella LT, Rothier EKC, Vilella OV, Ongkosuwito EM, Breuning KH. Virtual setup: application in orthodontic practice. J Orofac Orthop. 2016 Nov;77(6):409-19.

5. Westerlund A, Tancredi W, Ransjo M, Bresin A, Psonis S, Torgersson O: Digital casts in orthodontics. A comparison of 4 software systems. Am J Orthod Dentofacial Orthop 2015;147:509-16. doi: 10.1007/s00056-016-0048-y.

6. Bukhari SAA, Reddy KA, Reddy MR, Shah SH. Evaluation of virtual models (3Shape Ortho System) in assessing accuracy and duration of model analyses based on the severity of crowding. Saudi J Dent Res 2017;8(1-2):11-8. doi: 10.1016/j.sjdr.2016.05.004.

7. Mac Kriel EA. Accuracy of orthodontic digital study models [thesis]. Faculty of Dentistry, University of the Western Cape; 2012.

8. Fleming PS, Marinho V, Johal A. Orthodontic measurements on digital study models compared with plaster models: a systematic review. Orthod Craniofac Res. 2011 Feb;14(1):1-16. doi: 10.1111/j.1601-6343.2010.01503.x.

9. Luu NS, Nikolcheva LG, Retrouvey JM, Flores-Mir C, El-Bialy T, Carey JP, et al. Linear measurements using virtual study models. Angle Orthod. 2012 Nov;82(6):1098-106. doi: 10.2319/110311-681.1.

10. Rossini G, Parrini S, Castroflorio T, Deregibus A, Debernardi CL. Diagnostic accuracy and measurement sensitivity of digital models for orthodontic purposes: A systematic review. Am J Orthod Dentofacial Orthop. 2016 Feb;149(2):161-70. doi: 10.1016/j.ajodo.2015.06.029.

11. Michelinakis G, Apostolakis D, Tsagarakis A, Kourakis G, Pavlakis E. A comparison of accuracy of 3 intraoral scanners: A single-blinded in vitro study. J Prosthet Dent. 2020 Nov;124(5):581-8. doi: 10.1016/j.prosdent.2019.10.023.

12. Camardella LT, Breuning H, Vilella OV. Are there differences between comparison methods used to evaluate the accuracy and reliability of digital models? Dental Press J Orthod. 2017 Feb;22(1):65-74. doi: 10.1590/2177-6709.22.1.065-074.oar. 
13. Camardella LT, Ongkosuwito EM, Penning EW, KuijpersJagtman AM, Vilella OV, Breuning KH. Accuracy and reliability of measurements performed using two different software programs on digital models generated using laser and computed tomography plaster model scanners. Korean J Orthod. 2020 Jan;50(1):13-25. doi: 10.4041/kjod.2020.50.1.13.

14. Whetten JL, Williamson PC, Heo G, Varnhagen C, Major PW. Variations in orthodontic treatment planning decisions of Class II patients between virtual 3-dimensional models and traditional plaster study models. Am J Orthod Dentofacial Orthop. 2006 Oct;130(4):485-91. doi: 10.1016/j.ajodo.2005.02.022.

15. Okunami TR, Kusnoto B, BeGole E, Evans CA, Sadowsky C, Fadavi S. Assessing the American Board of Orthodontics objective grading system: digital vs plaster dental casts. Am J Orthod Dentofacial Orthop. 2007 Jan;131(1):51-6. doi: 10.1016/j.ajodo.2005.04.042.

16. Akyalcin S, Dyer DJ, English JD, Sar C. Comparison of 3-dimensional dental models from different sources: diagnostic accuracy and surface registration analysis. Am J Orthod Dentofacial Orthop 2013;144: 831-7.

17. Wiranto MG, Engelbrecht WP, Nolthenius HET, Meer WJ, Ren Y. Validity, reliability, and reproducibility of linear measurements on digital models obtained from intraoral and cone-beam computed tomography scans of alginate impressions. Am J Orthod Dentofacial Orthop. 2013 Dec;144(6):831-7. doi: 10.1016/j.ajodo.2013.08.014.

18. Leifert MF, Leifert MM, Efstratiadis SS, Cangialosi TJ. Comparasion of space analysis evaluations with digital models and plaster dental casts. Am J Orthod Dentofacial Orthop. 2009 Jul;136(1):16. e1-4; discussion 16. doi: 10.1016/j.ajodo.2008.11.019.

19. Santoro M, Galkin S, Teredesai M, Nicolay OF, Cangialosi TJ. Comparison of measurements made on digital and plaster models. Am J Orthod Dentofacial Orthop. 2003 Jul;124(1):101-5. doi: 10.1016/s0889-5406(03)00152-5.

20. Brusco N, Andreetto M, Lucchese L, Carmignato S, Cortelazzo GM. Metrological validation for 3D modeling of dental plaster casts. Med Eng Phys. 2007 Nov;29(9):954-66. doi: 10.1016/j.medengphy.2006.10.011.

21. Chiu A, Chen YW, Hayashi J, Sadr A. Accuracy of CAD/CAM Digital Impressions with Different Intraoral Scanner Parameters. Sensors (Basel). 2020 Feb 20;20(4):1157. doi: 10.3390/s20041157. 